\title{
Light Chain Distribution in Immune Deposits on Glomeruli of Kidneys in Human Renal Disease *
}

\author{
Roger C. Herdman, $\dagger$ Richard Hong, $\ddagger$ Alfred F. Michael, $\S$ and \\ Robert A. Good \| \\ (From the Pediatric Research Laboratories of the Variety Club Heart Hospital and the \\ Department of Microbiology, University of Minnesota, Minneapolis, Minn.)
}

\begin{abstract}
Summary. The $\gamma$ G-immunoglobulin (IgG) deposited on glomeruli of 32 percutaneous renal biopsies from 30 patients with various forms of renal disease was examined by fluorescent techniques to determine its light chain composition. Serum kappa: lambda light chain ratios were determined on 17 serum samples from 16 of the 30 patients.

Glomerular IgG staining with only antikappa antiserum, only antilambda antiserum, and with both antisera was demonstrated. No correlation of serum kappa: lambda light chain ratios with the type or types of light chains demonstrable on the glomeruli was observed. We concluded that the IgG deposited on glomeruli of patients with various forms of renal disease is not a nonspecific sample of the circulating immunoglobulin pool but may well reflect more specific selection, as in the deposition of specific antibody formed against exogenous or endogenous antigens during the development of the renal lesion.
\end{abstract}

\section{Introduction}

Human 7 S $\gamma$ G-immunoglobulins (IgG) are composed of dissimilar polypeptide chains of different molecular weights called heavy and light chains. The light chains are divided into two major antigenic types, Type I or kappa and Type II or lambda. Circulating IgG contains types kappa and lambda in approximately a $2: 1$ ratio (kappa: lambda) $(1,2)$, and specific antibodies also contain both types in varying ratios (3).

Recently Leddy and Bakemeier (4) investigated the light chain types of human IgG autoantibodies obtained from the surface of red cells of patients

* Submitted for publication June 6, 1966; accepted October 13, 1966.

Aided by grants from the U. S. Public Health Service (HE-05662, HE-06314, and HE-02085), National Foundation, and American Heart Association.

$\dagger$ Address requests for reprints to Dr. Roger C. Herdman, Dept. of Pediatrics, University of Minnesota Medical School, Minneapolis, Minn. 55455.

$\ddagger$ Recipient of a U. S. Public Health Service Career Research Development Award.

§ Established Investigator, American Heart Association.

II American Legion Memorial Heart Research Professor of Pediatrics and Microbiology. with autoimmune hemolytic anemia. In most cases these autoantibodies did not show the usual $2: 1$ ratio, but consisted of either type kappa or type lambda.

The localization of $\operatorname{IgG}$ and $\beta_{1 \mathrm{c}}$-globulin on the glomeruli of patients with various forms of glomerulonephritis suggests that immune mechanisms play a role in these diseases. Although the exact pathogenesis has not been defined, certain diseases, such as acute poststreptococcal glomerulonephritis, are similar morphologically to experimental antigen-antibody complex disease (5). The presence of IgG on the glomeruli could also reflect localization of antibody having specificity for the kidney itself, or might even represent deposition of aggregated $\gamma$-globulin similar to that occurring in the experimental animal (6).

To investigate further the mechanisms involved in the glomerular deposition of IgG in patients with diffuse renal disease, we determined the light chain types in these deposits by immunofluorescent methods. In addition, we estimated serum kappa : lambda ratios in half of these patients to correlate them with the kappa and lambda chain deposition in the kidney. We found that the glomerular 


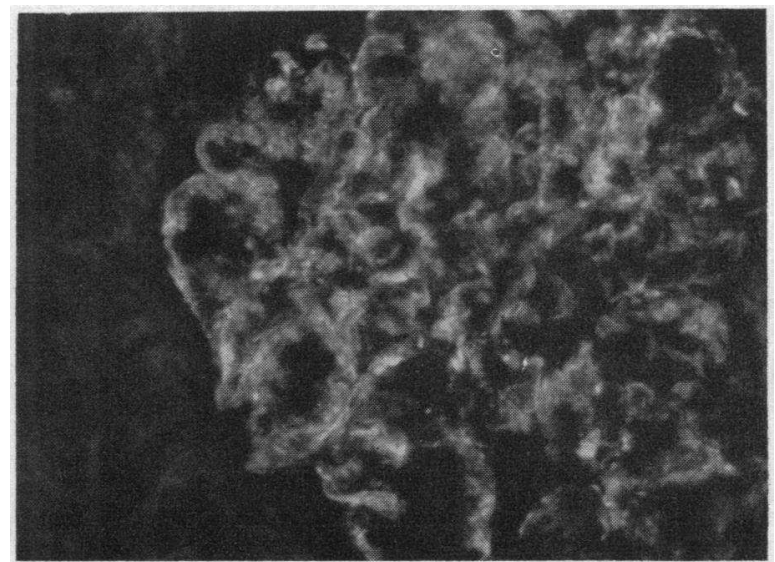

Fig. 1. FluORESCENCE PHOTOMICROGRAPH OF RENAL BIOPSY TISSUE OF A PATIENT WITH CHRONIC GLOMERULONEPHRITIS (PATIENT 29). A part of a glomerulus shows $3+$ linear and lumpy fluorescence due to IgG; $\times 250$.

deposition tended to be predominantly kappa or lambda and to be independent of the kappa: lambda ratio of the circulating immunoglobulin pool.

\section{Methods}

Human light chains of types kappa and lambda were obtained in the form of Bence Jones proteins from the urine of patients with multiple myeloma. ${ }^{1}$ The light chains were purified by gel filtration through Sephadex G-100 and by ion exchange chromatography with DEAE Sephadex A-50 according to the method of Bernier and Putnam (7). The isolated proteins gave single peaks and had observed sedimentation coefficients of 3.1 (kappa) and 2.8 (lambda) on analytical ultracentrifugation; single lines were seen on immunoelectrophoresis with rabbit antiserum to human $\operatorname{IgG}$ and whole human serum. Antisera against the kappa and lambda antigens were prepared by injecting $1.0 \mathrm{mg}$ of antigen emulsified in complete Freund's adjuvant ${ }^{2}$ into the heel pads and at multiple sites subcutaneously in rabbits. Intravenous boosters of $1.0 \mathrm{mg}$ were administered after 1 month, and the animals were bled 2 weeks later. The antisera obtained reacted on immunoelectrophoresis with the specific immunizing light chain and not with the other light chain or heavy chains. Single lines were also obtained when the antisera were reacted against whole human sera.

Kidney tissue used in this investigation was obtained by percutaneous kidney biopsy primarily of patients hospitalized on the Pediatric and Internal Medicine Services of the University of Minnesota Hospitals. ${ }^{3}$ The diagno-

1 The light chains were typed and the urine samples supplied through the courtesy of Dr. Ralph C. Williams, Jr., Dept. of Medicine, University of Minnesota School of Medicine.

2 Difco Laboratories, Detroit, Mich.

${ }^{3}$ Renal tissue was also obtained from Dr. John Wilson. sis of the renal disease in each patient was based on clinical and laboratory findings as well as routine microscopy of the renal biopsy. Only patients demonstrating IgG and $\beta_{1 \mathrm{c}}$-globulin on the glomeruli are included in this report.

The renal tissue was frozen by immersion in isopentane precooled in liquid nitrogen, sectioned in a Lipshaw cryostat at a thickness of $4 \mu$, and stained with fluorescein isothiocyanate-conjugated rabbit $\boldsymbol{\gamma}$-globulin containing antibodies to human IgG (anti-IgG) or to human $\beta_{1 \mathrm{C}}$ globulin (anti- $\beta_{\mathbf{1 C}}$ ) according to methods previously described (5). The possibility of occurrence of nonspecific fluorescence was controlled as previously reported (5). Tissue sections were also stained with fluorescein conjugated to rabbit $\boldsymbol{\gamma}$-globulin containing antibodies to kappa chains (antikappa) or lambda chains (antilambda). The majority of sections were stained at the same time. All sections were stained with the same antisera by the same person. Repeated staining of the same sections yielded the same results. In no case did sections that did not stain with anti-IgG stain with antikappa or antilambda. Freshly obtained tissue was used when possible and generally gave more intense staining than old frozen tissue. However, the slight fading that occurred on storage affected anti-IgG, antikappa, and antilambda equally. Quantitative precipitin curves revealed that antilambda was six times as active as antikappa. Because the staining technique used always involves a great excess of antiserum, this difference did not affect the intensity of staining. Nevertheless, the antilambda was diluted six times with isotonic saline in our work. Intensity of staining for IgG, kappa, and lambda chains as reported

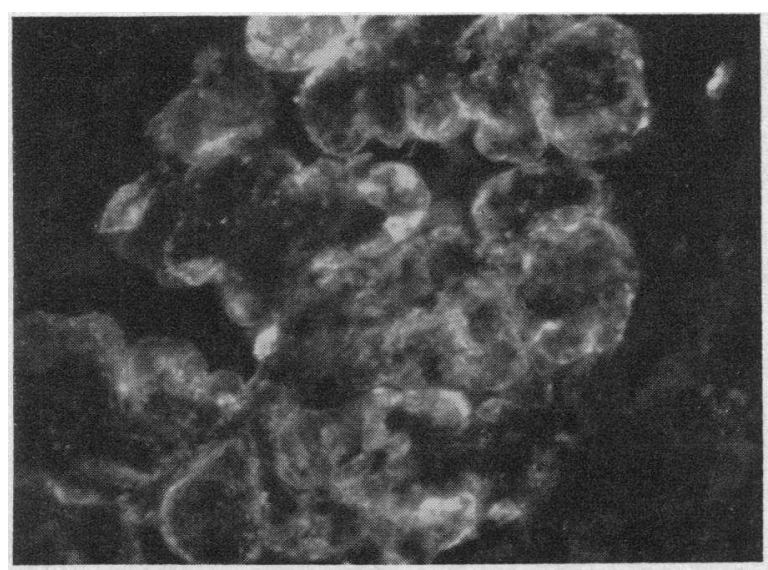

Fig. 2. FluORESCENCE PHOTOMICROGRAPH OF RENAL biopsy tissue of Patient 29: another glomerulus SHOWING THE SAME DISTRIBUTION OF FLUORESCENT MATERIAL AS IN Figure 1. There is $2+$ fluorescence due to kappa chains ; $\times 500$.

University of Utah Medical Center, Salt Lake City, Utah (Patients 10 and 24) ; Dr. John Burns, St. Paul, Minn. (Patients 17, 19, and 20); and Dr. R. K. Cutler, King County Hospital, Seattle, Wash. (Patients 26 and 28). 
TABLE I

Glomerular fluorescence and serum kappa: lambda ratios in human renal disease

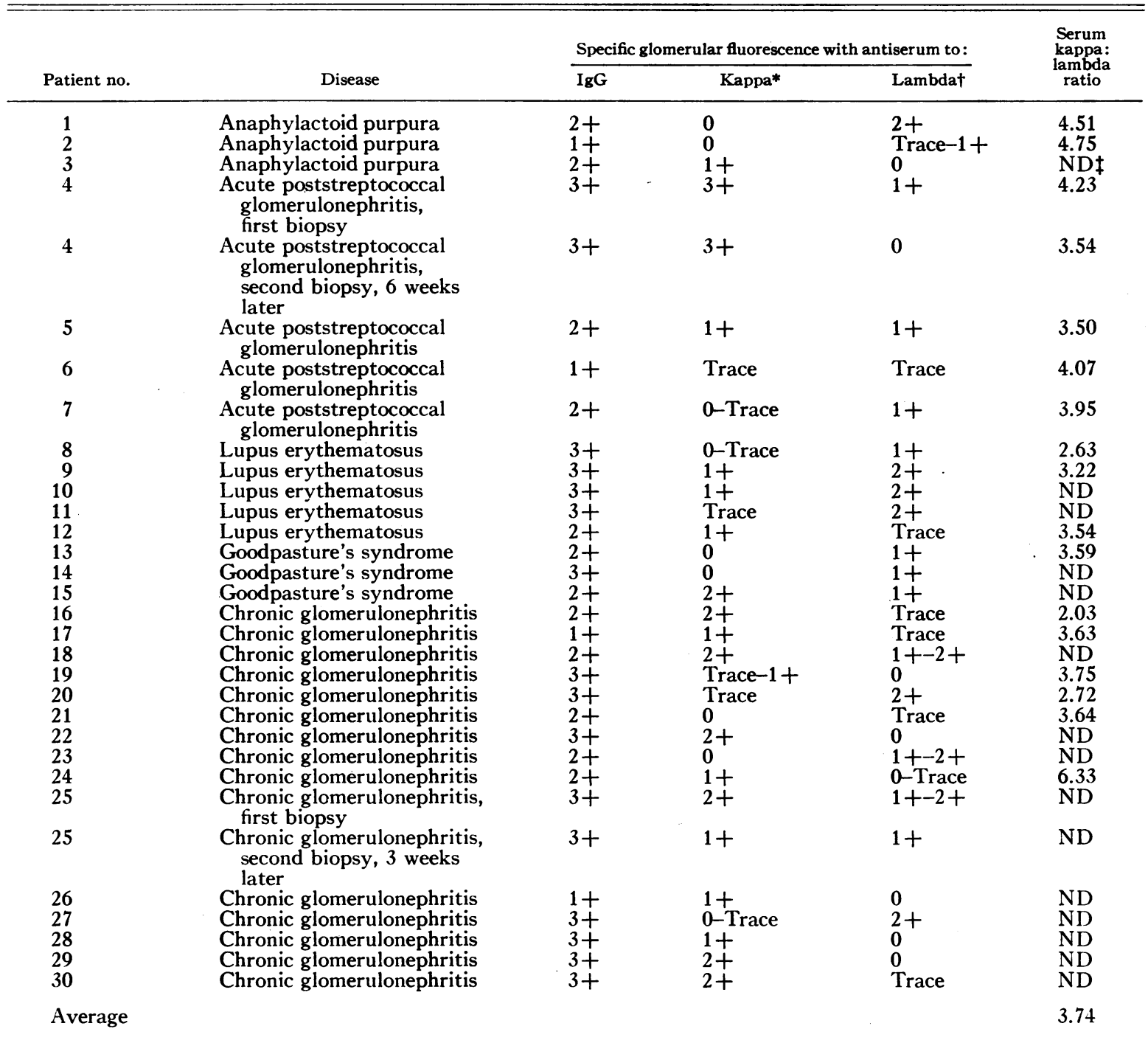

* Kappa = light chain kappa (Type I).

$\dagger$ Lambda = light chain lambda (Type II).

$\ddagger$ ND $=$ not done.

in Table I was estimated as 0 , trace, $1+, 2+$, and $3+$ by three observers independently. Those biopsies graded 0 had no apparent specific staining; those graded trace had only occasional areas of specific fluorescence located on a few of the glomeruli or extremely faint generalized fluorescence. These biopsies graded $1+$ had fluorescence intense enough to be photographed, those graded $2+$ had fluorescence of increasing intensity in increasing numbers of locations, and those graded $3+$ had brilliant generalized fluorescence.

Serum levels of kappa and lambda chains were determined in duplicate in Oudin tubes with antikappa or antilambda incorporated in agar, and the patient's se- rum was layered on top. The distance traveled by the precipitin band was compared with distances on a standard curve prepared at the same time with purified light chains of each type in concentrations established by absorption at $280 \mathrm{~m} \mu$ in the Beckman spectrophotometer. These determinations as routinely performed in our laboratory are accurate within $10 \%$.

\section{Results}

The 30 patients with renal disease whose biopsies showed demonstrable deposition of IgG and 


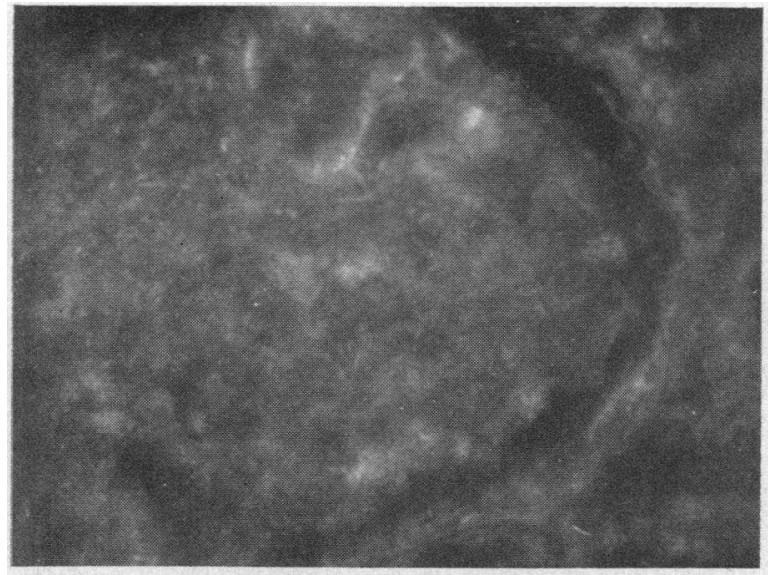

Fig. 3. FluORESCENCE PHOTOMICROGRAPH OF RENAL biopsy tissue of Patient 29: a glomerulus stained WITH RABBIT ANTISERUM TO HUMAN LAMBDA CHAINS. No significant fluorescent material is present $\times 250$.

$\beta_{1 \mathrm{C}}$-globulin are listed in Table I, grouped by disease. The results of the fluorescent staining for IgG, kappa chains, and lambda chains are tabulated, as is the serum kappa : lambda ratio.

Sixteen biopsies had an intensity of fluorescent staining in the glomeruli that was greater with antikappa than with antilambda, and in 7 of these there was no demonstrable fluorescence with antilambda (Figures 1-3). Thirteen biopsies had fluorescent staining that was greater with antilambda than with antikappa, and 6 of them showed no significant kappa staining. Three biopsies had approximately equal fluorescence with both antisera. In no case was the intensity of staining with fluorescein-conjugated antilight chain antisera greater than the intensity of staining with antiIgG, and in most cases it was somewhat less. There was no correlation between the type of renal disease and the type of light chain staining. Since no difference in location of fluorescent material could be found with any of the antisera that gave staining of sufficient intensity, it appeared likely that the same immune deposits were being stained by the different antisera. The fluorescence changed somewhat in the two patients who had serial biopsies. In Patient 4, the initial biopsy stained both with antikappa and antilambda (Figure 4), but in the second biopsy (Figures 5 and 6 ) there was no lambda chain fluorescence although the glomeruli retained their fluorescence with antikappa. In Patient 25 the fluorescence for both kappa and lambda chains persisted in the second biopsy but had diminished slightly.

The serum kappa-lambda ratios showed no correlation with the degree of fluorescence observed on the kidney when antikappa or antilambda sera were used, nor was there any correlation with the type of renal disease. The kappa: lambda ratios obtained in this study are significantly higher than normal values previously reported, but are similar to those observed in similar diseases (2). The average serum kappa: lambda ratio for the group as a whole was 3.74. The average kappa: lambda ratio of the serum from patients whose biopsies showed predominantly fluorescent staining for kappa chains was 3.88. For those whose biopsies showed predominantly fluorescent staining for lambda chains, the average kappa: lambda ratio was 3.63, and for those with approximately equal kappa and lambda staining the value was 3.79 . These ratios are not significantly different.

\section{Discussion}

The immunoglobulin deposited on basement membranes in experimental renal disease has been shown to be antibody complexed with antigen and complement, heterologous antikidney antibody, or antibody directed against antigen bound to glomerular basement membrane (8-11).

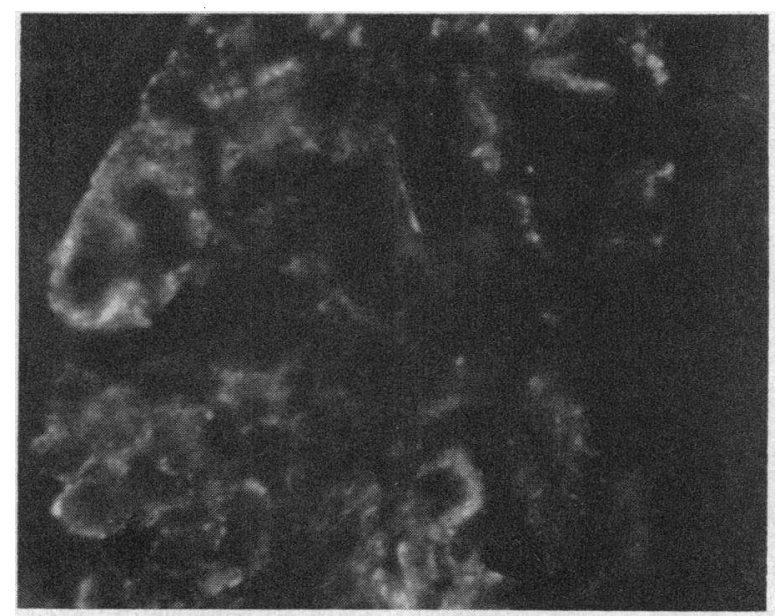

Fig. 4. Fluorescence PHOtomicrograph OF RENAL BIOPSY TISSUE OF A PATIENT WITH ACUTE POSTSTREPTOCOCCAL GLOMERULONEPHRITIS (PATIENT 4) : FIRST BIOPSY, SHOWING $1+$ FLUORESCENCE DUE TO LAMBDA CHAINS. $\times 250$. 
Evidence has been presented that in certain forms of human glomerulonephritis specific antibody may be important in the initiation and perpetuation of the disease. This evidence includes the fixation of complement with immunoglobulin to the glomerular basement membrane, low levels of serum complement, and evidence of complement utilization in some renal diseases (12); the selective deposition of IgG and not IgM or IgA on the glomeruli; and the disappearance of immunoglobulin deposits correlated with improved renal function after prolonged immunosuppressive therapy (13) or associated with spontaneous improvement or recovery (5). To date, however, although circulating antikidney antibodies have been found in various forms of human renal disease, in no case has IgG bound to human glomeruli been identified as antikidney antibody; nor has any glomerular IgG been identified as specific antibody except the antinuclear antibody deposited on glomerular basement membranes of patients with lupus erythematosus (14). Michael, Drummond, Vernier, and Good suggested the possibility that the IgG on glomeruli of patients with acute poststreptococcal glomerulonephritis might be aggregates of $\gamma$-globulin from the general circulating pool and not antibody directed specifically against any particular antigen (10). However, the data presented in this report indicate that the IgG on the glomeruli of patients with various forms of glomerulonephritis has a different kappa and lambda composition from that of the immunoglobulin pool

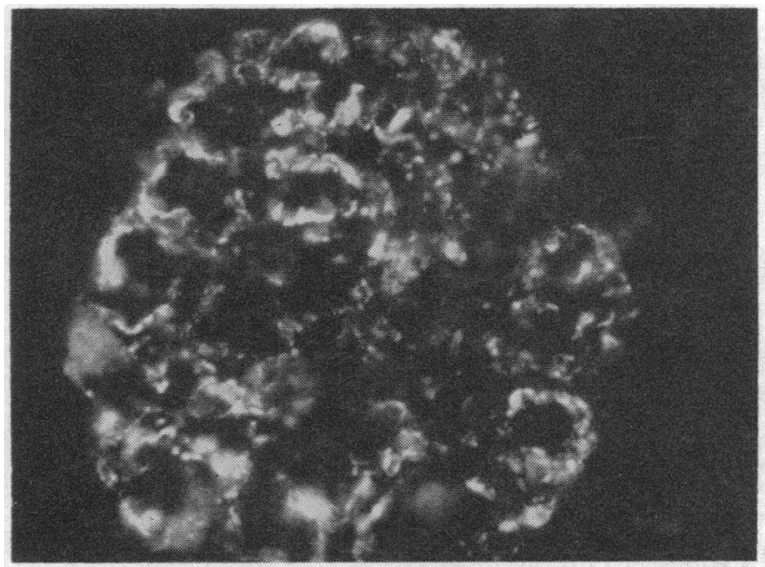

Fig. 5. FluORESCENCE PHOTOMICROGRAPH OF RENAL BIOPSY tisSUe OF PATIENT 4: SECOND BIOPSY, SHOWING HEALING PHASE. This glomerulus shows $3+$ lumpy fluorescence due to IgG; $\times 250$.

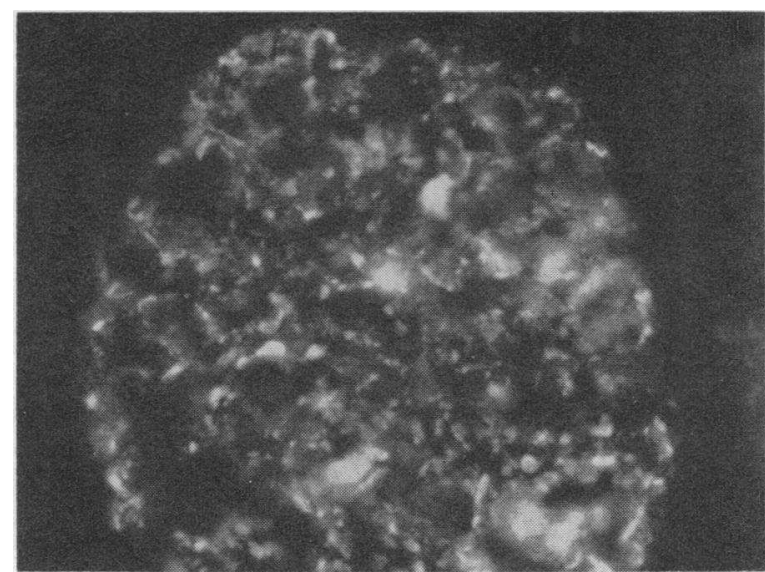

Fig. 6. Fluorescence PHOTOMICROGRAPH. OF RENAL biopsy tissue of Patient 4: a glomerules with $3+$ FLUORESCENCE DUE TO KAPPA CHAINS. The distribution of fluorescent material is similar to that due to IgG. For brevity, the glomerulus showing no fluorescent staining for lambda chains is omitted; $\times 250$.

of the peripheral blood. This observation carries the implication that the IgG located on the glomerular tuft of the kidney does not involve nonspecific deposition of immunoglobulin from the immunoglobulin pool, but rather that the immunoglobulin on the glomerular membranes is derived from antibody. This is especially clear in the cases of anaphylactoid purpura nephritis and Goodpasture's syndrome, in which, with one exception, there was staining with antisera directed against only one of the light chains. Absence of fluorescent staining for a particular antigen in a tissue may be due to absence of the antigen, its presence in quantities too small to be detected by immuno. fluorescent methods, or the lack of availability of antigenic sites to react with the specific fluoresceinconjugated antibody. Since there is no evidence in human renal disease for the third possibility, we have concluded that the lack of staining indicates that the antigen is not present at all or is present in very minute amounts. The demonstration of one light chain and not the other in the deposited IgG is presumptive evidence that this IgG is a special part rather than a nonspecific sample of the host IgG. Since individual plasma cells are known to produce $\gamma$-globulin of only one light chain type (15), it is possible that a clone of cells and its descendants may have been selectively stimulated to produce the specific $\operatorname{IgG}$ seen on the kidney at any particular time. 


\section{References}

1. Mannik, M., and H. G. Kunkel. Two major types of normal 7S $\gamma$-globulin. J. exp. Med. 1963, 117, 213.

2. McKelvey, E. M., and J. L. Fahey. Immunoglobulin changes in disease: quantitation on the basis of heavy polypeptide chains, IgG $(\gamma \mathrm{G})$, IgA $(\gamma \mathrm{A})$, and $\operatorname{IgM}(\gamma \mathrm{M})$, and of light polypeptide chains, Type K (I) and Type L (II). J. clin. Invest. 1965, 44, 1778.

3. Mannik, M., and H. G. Kunkel. Localization of antibodies in group I and group II $\boldsymbol{\gamma}$-globulins. J. exp. Med. 1963, 118, 817.

4. Leddy, J. P., and R. F. Bakemeier. Structural aspects of human erythrocyte autoantibodies. I. L chain types and electrophoretic dispersion. J. exp. Med. 1965, 121, 1.

5. Michael, A. F., Jr., K. N. Drummond, R. A. Good, and R. L. Vernier. Acute poststreptococcal glomerulonephritis: immune deposit disease. J. clin. Invest. 1966, 45, 237.

6. Fish, A. J., and A. F. Michael. Glomerular localization of aggregated proteins. Fed. Proc. 1966, 25, 231.

7. Bernier, G. M., and F. W. Putnam. Polymerism, polymorphism, and impurities in Bence-Jones proteins. Biochim. biophys. Acta (Amst.) 1964, 86, 295.
8. Dixon, F. J., J. J. Vazquez, W. O. Weigle, and C. G. Cochrane. Pathogenesis of serum sickness. Arch. Path. 1958, 65, 18.

9. Mellors, R. C. Histochemical demonstration of the in vivo localization of antibiodies. Antigenic components of the kidney and the pathogenesis of glomerulonephritis. J. Histochem. Cytochem. 1955, 3, 284.

10. Michael, A. F., K. N. Drummond, R. L. Vernier, and R. A. Good. Immunologic basis of renal disease. Pediat. Clin. N. Amer. 1964, 11, 685.

11. Heymann, W., D. B. Hackel, S. Harwood, S. G. F. Wilson, and J. L. P. Hunter. Production of nephrotic syndrome in rats by Freund's adjuvants and rat kidney suspensions. Proc. Soc. exp. Biol. (N. Y.) 1959, 100, 660.

12. Gewurz, H., R. J. Pickering, and R. A. Good. In preparation.

13. Michael, A. F., R. L. Vernier, K. N. Drummond, J. I. Levitt, R. C. Herdman, A. J. Fish, and R. A. Good. Immunosuppressive therapy of chronic renal disease. New Engl. J. Med. 1967, in press.

14. Freedman, P., and A. S. Markowitz. Isolation of antibody-like gamma-globulin from lupus glomeruli. Brit. med. J. 1962, 1, 1175.

15. Bernier, G. M., and J. J. Cebra. Frequency distribution of $\alpha, \gamma, \kappa$ and $\lambda$ polypeptide chains in human lymphoid tissues. J. Immunol. 1965, 95, 246.

\section{ANNOUNCEMENT OF MEETINGS}

The American Federation for Clinical Research will hold its Twentyfourth Annual Meeting in Atlantic City, N. J., in the Pennsylvania Room, Haddon Hall, on Sunday, April 30, 1967, at 9:00 a.m. Joint sectional meetings with The American Society for Clinical Investigation will be held on Sunday afternoon at Chalfonte-Haddon Hall, and additional meetings sponsored by The American Federation for Clinical Research will be held on Sunday evening.

The American Society for Clinical Investigation, Inc., will hold its Fifty-ninth Annual Meeting in Atlantic City, N. J., on Monday, May 1, at 9:00 a.m., in the Pennsylvania Room, Haddon Hall, and will join The American Federation for Clinical Research in simultaneous sectional meetings on Sunday afternoon, April 30, at Chalfonte-Haddon Hall.

The Association of American Physicians will hold its Eightieth Annual Meeting in Atlantic City, N. J., in the Pennsylvania Room, Haddon Hall, on Tuesday, May 2, at 9:30 a.m., and in the Vernon Room, on Wednesday, May 3, at 9:30 a.m. 